\title{
THE SPECTRA OF COMPACT OPERATORS IN HILBERT SPACES
}

\author{
by T. T. WEST
}

(Received 5 May, 1964)

1. Introduction. In [2] a condition, originally due to Olagunju, was given for the spectra of certain compact operators to be on the real axis of the complex plane. Here, by using conformal mappings, this result is extended to more general curves. The problem divides naturally into two cases depending on whether or not the curve under consideration passes through the origin. Discussion is confined to the prototype curves $C_{0}$ and $C_{1}$. The case of $C_{0}$, the unit circle of centre the origin, is considered in $\S 3$; this problem is a simple one as the spectrum is a finite set. In $\S 4$ results are given for $C_{1}$, the unit circle of centre the point 1 , and some results on ideals of compact operators, given in $\S 2$, are needed. No attempt has been made to state results in complete generality (see [2]); this paper is kept within the framework of Hilbert space, and particularly simple conditions may be given if the operators are normal.

This work was undertaken while the author held an 1851 Overseas Scholarship. He wishes to thank Dr F. Smithies for suggesting the problem and for his advice and encouragement.

2. Preliminary results. If $H$ is a Hilbert space, let $\mathscr{B}(H)$ be the algebra of bounded linear operators in $H$. If $T \in \mathscr{B}(H)$, write $T^{*}$ for the Hilbert adjoint of $T . \mathscr{C}(H)$ will be the ideal of compact operators in $H$. Let $T \in \mathscr{C}(H)$ and denote by $\sigma_{0}(T)=\left(\lambda_{j}\right)$ the non-zero eigenvalues of $T$ repeated according to algebraic multiplicity. $T^{*} T$ is a positive operator in $\mathscr{C}(H)$ and therefore possesses a unique square $\operatorname{root}\left(T^{*} T\right)^{\frac{1}{2}}$ which is also in $\mathscr{C}(H)$. Write $\sigma_{0}\left(\left(T^{*} T\right)^{\frac{1}{t}}\right)=\left(\mu_{j}\right)$.

If $p \geqq 1, \mathscr{C}_{p}(H)$ will denote the class of operators $T \in \mathscr{C}(H)$ for which $\sum_{j} \mu_{j}^{p}<\infty$. Results of $\left[1\right.$, Vol. II, Sections X. 9 and X. 10] show that, for each $p, \mathscr{C}_{p}(H)$ is a two-sided ideal of $\mathscr{B}(H)$, and that

$$
\sum_{j}\left|\lambda_{j}\right|^{p} \leqq \sum_{j} \mu_{j}^{p}
$$

for $T \in \mathscr{C}_{p}(H) . \mathscr{C}_{1}$ is the trace class and $\mathscr{C}_{2}$ the Hilbert-Schmidt class of operators in $H$.

If $T \in \mathscr{C}_{1}(H)$ and $\left(\phi_{j}\right)$ is a complete orthonormal system in $H$, the trace of $T$ is defined by

$$
\operatorname{tr}\{T\}=\sum_{j}\left(T \phi_{j}, \phi_{j}\right)
$$

This series converges and is independent of the system $\left(\phi_{j}\right)[5$, p. 353, Theorem $1 ; 3$, p. 37, Lemma 1]. If $T \in \mathscr{C}_{p}(H)$ for some $p \geqq 1$, it follows from [4, Theorem 3] that $T^{n} \in \mathscr{C}_{1}(H)$ for integral $n \geqq p$; hence $\operatorname{tr}\left\{T^{n}\right\}$ exists for $n \geqq p$ and is equal to $\sum_{j} \lambda_{j}^{n}$.

If $T \in \mathscr{C}(H)$ and $\lambda$ is an isolated point of $\sigma(T)$, let $P(\lambda ; T)$ denote the spectral projection associated with $\lambda$. We have the formula

$$
P(\lambda ; T)=\frac{1}{2 \pi i} \int_{\gamma}(z I-T)^{-1} d z,
$$


where $\gamma$ is a circle of centre $\lambda$ containing no other points of $\sigma(T)$. If $\lambda$ is non-zero, $P(\lambda ; T)$ will have a finite dimensional range [1, Vol. I, Chap. VII $]$.

Our basic result comes from [2]. A polynomial $p$ will be called a complex polynomial if its coefficients are complex numbers; similarly for a real polynomial $q . R$ will denote the real axis of the complex plane and $R^{+}$the non-negative real axis. If $n$ is a strictly positive integer, we shall denote the hypothesis

$$
\text { " } \operatorname{tr}\left\{T^{n}\left(q(T)^{2}\right\} \text { is real and non-negative for all real polynomials } q "\right.
$$

by the symbol $Q(n, T)$.

THEOREM 2.1. If $T$ is a linear operator in a finite dimensional linear space, then

(i) $\sigma(T) \subset R$ if and only if $Q(n, T)$ holds for some even $n$;

(ii) $\sigma(T) \subset R^{+}$if and only if $Q(n, T)$ holds for some odd $n$.

Proof. This follows immediately from Theorems 3.1 and 4.2 of [2], on observing that any complex polynomial $p$ can be written

where $q$ and $r$ are real polynomials.

$$
p=q+i r
$$

Remark. Provided that $T$ satisfies certain conditions, this result may be given in the infinite dimensional case (see [2, Theorem 4.3]).

3. Circles with centre the origin. From now on we shall take $H$ to be infinite dimensional; the finite dimensional cases are straightforward. If $T \in \mathscr{C}(H)$ and $\sigma_{0}(T) \subset C_{0}, \sigma_{0}(T)$ is a finite set and we write $\left(\lambda_{1}, \ldots, \lambda_{k}\right)$ for the distinct members of $\sigma_{0}(T)$. Then zero is an isolated point of $\sigma(T)$ and we can form the spectral projection $P(0 ; T)$. The residue theorem shows that

and the projection

$$
I=P(0 ; T)+\sum_{j=1}^{k} P\left(\lambda_{j} ; T\right)
$$

$$
P=I-P(0 ; T)
$$

is of finite rank. A rotation will ensure that $1 \notin \sigma(T)$; then write

$$
S=f(T)=i(I+T)(I-T)^{-1} ;
$$

and denote by $\Gamma_{0}$ the arc of $C_{0}$ in the lower half-plane.

THEOREM 3.1. If $T \in \mathscr{C}(H)$ and $1 \notin \sigma(T)$, then

(i) $\sigma_{0}(T) \subset C_{0}$ if and only if $Q(n, P S)$ holds for some even $n$;

(ii) $\sigma_{0}(T) \subset \Gamma_{0}$ if and only if $Q(n, P S)$ holds for some odd $n$.

Proof. If $\sigma(T)$ is a finite set, the projection $P$ defined by (1) exists and is of finite rank. The one-one transformation

$$
w=f(z)=i(1+z)(1-z)^{-1}
$$


maps $C_{0}, \Gamma_{0}$ in the $z$-plane onto $R, R^{+}$in the $w$-plane (respectively). We have

and

$$
\begin{aligned}
& \sigma(T)=\left(\lambda_{j}\right) \cup(0), \\
& \sigma(S)=\left(f\left(\lambda_{j}\right)\right) \cup(i),
\end{aligned}
$$

$$
P(S ; f(\lambda))=P(T ; \lambda) \quad(\lambda \in \sigma(T))
$$

by the spectral mapping theorem [1, Vol. I, Chap. VII]. Hence

$$
\sigma(P S)=\left(f\left(\lambda_{j}\right)\right) \cup(0) \text {, }
$$

and the operator PS is of finite rank; hence its trace exists. Observing that $\sigma_{0}(T) \subset C_{0}, \Gamma_{0}$ (resp.) if and only if $\sigma(P S) \subset R, R^{+}$(resp.), we complete the proof by applying Theorem 2.1 to the operator $P S$.

Remark. The projection $P$ may be calculated from the formula

$$
P=I-\frac{1}{2 \pi i} \int_{\gamma}(z I-T)^{-1} d z,
$$

where $\gamma$ is a sufficiently small circle with centre the origin.

For the case of a compact normal operator we shall need the following lemmas.

LEMMA 3.2. If $\left(t_{1}, \ldots, t_{n}\right)$ is a set of non-zero real numbers such that

$$
\sum_{j=1}^{n} t_{j}=n
$$

and

$$
\sum_{j=1}^{n} t_{j}^{2}=n
$$

then $t_{j}=1(1 \leqq j \leqq n)$.

Proof. (3) may be regarded as being the equation of a sphere of centre zero and radius $\sqrt{ } n$ in $n$ dimensional Euclidean space. Then (2) is the equation of the tangent plane at the point $(1,1, \ldots, 1)$ on the sphere and this point of contact is unique.

LEMMA 3.3. If $\left(t_{j}\right)_{j=1}^{\infty}$ is a set of non-negative real numbers and if

$$
\sum_{j=1}^{\infty} t_{j}^{k}=p \quad(k=1,2, \ldots)
$$

where $p \geqq 0$, then only a finite number of the $t_{j}$ 's are non-zero and these are all equal to one.

Proof. If, for any $j, t_{j}>1$, by choice of $k$ we can make

$$
\sum_{j=1}^{\infty} t_{j}^{k}>p,
$$

which contradicts the hypothesis. Hence $t_{j} \leqq 1$ for each $j$. Now

$$
\sum_{1}^{\infty} t_{j}-\sum_{1}^{\infty} t_{j}^{2}=0
$$


i.e.

$$
\sum_{i}^{\infty} t_{j}\left(1-t_{j}\right)=0
$$

and the individual terms on the left side of (4) are non-negative; hence they must all be zero and $t_{j}=0$ or 1 for each $j$. It follows that $p$ is an integer and that $p$ of the $t_{j}$ 's are one and the rest zero.

THEOREM 3.4. If $T$ is a compact normal operator in $H$, the following conditions are each necessary and sufficient for $\sigma_{0}(T) \subset C_{0}$ :

(i) the non-zero eigenvalues of $T$, repeated according to multiplicity, are $n$ in number, and

(ii) $\operatorname{tr}\left\{T^{*} T\right\}$ exists, and

$$
\operatorname{tr}\left\{T^{*} T\right\}=\operatorname{tr}\left\{\left(T^{*} T\right)^{2}\right\}=n ;
$$

$$
\operatorname{tr}\left\{\left(T^{*} T\right)^{k}\right\}=p \quad(k=1,2, \ldots)
$$

where $p \geqq 0$.

Proof. In both cases, the necessity of the condition is obvious. To prove the sufficiency, we note that, since $\operatorname{tr}\left\{T^{*} T\right\}$ exists,

$$
\operatorname{tr}\left\{\left(T^{*} T\right)^{k}\right\}=\sum_{j}\left|\lambda_{j}\right|^{2 k}
$$

and the results follow from Lemmas 3.2 and 3.3 with $t_{j}=\left|\lambda_{j}\right|^{2}$.

As the next theorem shows, these operators have a simple structure.

THEOREM 3.5. If $T$ is a compact normal operator in $H$, then $\sigma_{0}(T) \subset C_{0}$ if and only if there is a finite dimensional subspace $M$ of $H$ such that $M$ and its orthogonal complement $M^{\perp}$ are invariant under $T, T$ restricted to $M$ is unitary and $T$ restricted to $M^{\perp}$ is zero.

Proof. If $\sigma_{0}(T) \subset C_{0}$, then $\sigma_{0}(T)=\left(\lambda_{j}\right)_{1}^{n}$, and

$$
T=\sum_{j=1}^{n} \lambda_{j} \phi_{j} \otimes \Phi_{j}
$$

using the notation of [3], where $\left(\phi_{j}\right)_{1}^{n}$ is an orthonormal system. Let the subspace spanned by $\left(\phi_{j}\right)_{j=1}^{n}$ be $M$. Then $M$ is invariant under $T$ and the restriction of $T$ to $M$ is unitary. If $\psi \in M^{\perp}$,

$$
\left(\psi, \phi_{j}\right)=0 \quad(j=1, \ldots, n)
$$

hence $T \psi=0$, and $T$ restricted to $M^{\perp}$ is the zero operator.

The converse is obvious; hence the proof is complete.

4. Circles passing through the origin. The problem of a circle of this type may be reduced to the case of $C_{1}$ by a suitable rotation and magnification. Let us denote by $\Gamma_{1}$ the arc of $C_{1}$ in the lower half-plane and write

if $2 \notin \sigma(T)$.

$$
S=f(T)=i T(2 I-T)^{-1}
$$


THEOREM 4.1. If $T \in \mathscr{C}_{p}(H)$ for some $p \geqq 1$ and $2 \notin \sigma(T)$, then

(i) $\sigma(T) \subset C_{1}$ if and only if $Q(n, S)$ holds for some even $n \geqq p$;

(ii) $\sigma(T) \subset \Gamma_{1}$ if and only if $Q(n, S)$ holds for some odd $n \geqq p$.

Proof. The one-one transformation

$$
w=f(z)=i z(2-z)^{-1}
$$

maps $C_{1}, \Gamma_{1}$ in the $z$-plane onto $R, R^{+}$in the $w$-plane, respectively. If $\sigma_{0}(T)=\left(\lambda_{j}\right)$, the spectral mapping theorem implies that $\sigma_{0}(S)=(f(\lambda))$; hence $\sigma(T) \subset C_{1}, \Gamma_{1}$ if and only if $\sigma(S) \subset R, R^{+}$respectively.

Now $S \in \mathscr{C}_{p}(H)$; thus $\operatorname{tr}\left\{S^{n}\right\}$ exists for integral $n \geqq p$ and the theorem is proved by applying the infinite dimensional version of Theorem 2.1 to the operator $S$.

If $2 \in \sigma(T)$ let $P(2 ; T)$ be the associated spectral projection and write $\sigma_{2}(T)$ for $\sigma(T)$ with the eigenvalues equal to 2 omitted. If

$$
Q=I-P(2 ; T)
$$

we have $\sigma(Q T)=\sigma_{2}(T)$, and $Q T \in \mathscr{C}_{p}(H)$. The result for this case is obtained by putting $S=f(Q T)$ in Theorem 4.1.

Again, if $T$ is a compact normal operator, we get a simple condition.

THEOREM 4.2. If $T$ is a compact normal operator in $H, \sigma(T) \subset C_{1}$ if and only if

$$
T^{*} T=T^{*}+T \text {. }
$$

Proof. Let $\sigma_{0}(T)=\left(\lambda_{j}\right)$; then there is an orthonormal system $\left(\phi_{j}\right)$ in $H$ such that

and

$$
\begin{aligned}
T & =\sum \lambda_{j} \phi_{j} \otimes \bar{\phi}_{j}, \\
T^{*} & =\sum \lambda_{j} \phi_{j} \otimes \bar{\phi}_{j}, \\
T^{*} T & =\sum \lambda_{j} \lambda_{j} \phi_{j} \otimes \bar{\phi}_{j},
\end{aligned}
$$

$$
T^{*}+T=\sum\left(\lambda_{j}+\lambda_{j}\right) \phi_{j} \otimes \bar{\phi}_{j}
$$

Thus the hypothesis is equivalent to

i.e.

$$
\lambda_{j} \lambda_{j}=\lambda_{j}+\lambda_{j} \quad(j=1,2, \ldots)
$$

$$
\left|\lambda_{j}-1\right|=1 \quad(j=1,2, \ldots) \text {. }
$$

\section{REFERENCES}

1. N. Dunford and J. T. Schwartz, Linear operators (New York, 1962).

2. P. A. Olagunju and T. T. West, The spectra of Fredholm operators in locally convex spaces, Proc. Cambridge Philos. Soc. 60 (1964), 801-806.

3. R. Schatten, Norm ideals of completely continuous operators (Berlin, 1960).

4. C. Visser and A. C. Zaanen, On the eigenvalues of compact linear transformations, Nederl. Akad. Wetensch. Proc. Ser. A, 55 (1952), 71-78.

5. A. C. Zaanen, Linear analysis (Amsterdam, 1956).

\section{UNIVERSITY OF GLASGOW}

GLASGOW, W.2 